\title{
A taxonomic revision of Melanoxerus (Rubiaceae), with descriptions of three new species of trees from Madagascar
}

\author{
Kent Kainulainen
}

\begin{abstract}
KAINULAINEN, K. (2021). A taxonomic revision of Melanoxerus (Rubiaceae), with descriptions of three new species of trees from Madagascar. Candollea 76: 105-116. In English, English and French abstracts. DOI: http://dx.doi.org/10.15553/c2021v761a11

This paper provides a taxonomic revision of Melanoxerus Kainul. \& Bremer (Rubiaceae) - a genus of deciduous trees with eye-catching flowers and fruits that is endemic to Madagascar. Descriptions of three new species, Melanoxerus antsirananensis Kainul., Melanoxerus atropurpureus Kainul., and Melanoxerus maritimus Kainul. are presented along with distribution maps and a species identification key. The species distributions generally reflect the ecoregions of Madagascar, with Melanoxerus antsirananensis being found in the dry deciduous forests of the north; Melanoxerus atropurpureus in the inland dry deciduous forests of the west; Melanoxerus maritimus in dry deciduous forest on coastal sands; and Melanoxerus suavissimus (Homolle ex Cavaco) Kainul. \& B. Bremer in the dry spiny thicket and succulent woodlands of the southwest.
\end{abstract}

\section{Résumé}

KAINULAINEN, K. (2021). Révision taxonomique du genre Melanoxerus (Rubiaceae), avec la description de trois nouvelles espèces d'arbres de Madagascar. Candollea 76: 105-116. En anglais, résumés anglais et français. DOI: http://dx.doi.org/10.15553/c2021v761a11

Cet article propose une révision taxonomique de Melanoxerus Kainul. \& Bremer (Rubiaceae), un genre d'arbres à feuilles caduques avec des fleurs et des fruits attrayants qui est endémique de Madagascar. La description de trois nouvelles espèces, Melanoxerus antsirananensis Kainul., Melanoxerus atropurpureus Kainul. et Melanoxerus maritimus Kainul. est présentée accompagné de cartes de répartition et d'une clé d'identification des espèces. La répartition des espèces reflète généralement les écorégions de Madagascar: Melanoxerus antsirananensis pousse dans les forêts sèches de feuillus du nord; Melanoxerus atropurpureus dans les forêts de feuillus sèches de l'intérieur des terres de l'ouest; Melanoxerus maritimus dans la forêt sèche décidue sur sables côtiers; et Melanoxerus suavissimus (Homolle ex Cavaco) Kainul. \& B. Bremer dans le fourré épineux sec et les forêts succulentes du sud-ouest.

\section{Keywords}

RUBLACEAE - Gardenieae - Euclinia - Melanoxerus - Madagascar - Taxonomy - New species

Address of the author:

Gothenburg Botanical Garden, Carl Skottbergs Gata 22A, 41319 Gothenburg, Sweden. E-mail: kent.kainulainen@vgregion.se 


\section{Introduction}

The genus Melanoxerus Kainul. \& B. Bremer is part of the tribe Gardenieae in the flowering plant family Rubiaceae. It was segregated from Euclinia Salisb. based on the results of the molecular phylogenetic analyses by KaInUlainen \& BREMER (2014). Whereas Euclinia is restricted to central Africa, Melanoxerus is endemic to Madagascar, where it is widely distributed in the subarid to dry southern and western regions but also occurs in the dry to subhumid north. Melanoxerus is a genus of deciduous or semi-deciduous trees with smooth flaky bark and sympodial plagiotropic branches with terminally clustered leaves and scarious stipules covering the terminal buds. The flowers are showy and very fragrant, and the large fleshy fruits are lenticellate with conspicuous patterns. The genus can be further diagnosed in that leaves and flowers turn black when drying. It is readily distinct from the other genera of tribe Gardenieae in Madagascar, i.e., Catunaregam Wolf, Gardenia Ellis, and Hyperacanthus E. Mey. ex Bridson. Melanoxerus lacks the opposite-decussate spines of Catunaregam, the sheathing persistent stipules and resinous exudate of Gardenia, and the flowers and fruits are terminal, whereas in Hyperacanthus these appear axillary (Schatz, 2001; Madagascar Catalogue, 2021).

A taxonomic revision of Melanoxerus is warranted. Melanoxerus suavissimus (Homolle ex Cavaco) Kainul. \& B. Bremer is currently the only recognized species in the genus. It was originally described by CAvaco (1967) as Gardenia suavissima Homolle ex Cavaco, but shortly thereafter transferred to Euclinia by Leroy (1974). In the protologue, Cavaco (1967) lists several paratypes from western Madagascar that do not correspond to the same species as the holotype from Ambovombe in southernmost Madagascar. The populations from northern Madagascar are also morphologically distinct. Since plants of Melanoxerus are deciduous and often flower before the leaves flush, specimens collected at different times of the year can be difficult to match up, and this may in part explain how the new species presented in this paper have escaped taxonomic attention in the past despite their eyecatching flowers and fruits. In this study I recognize a total number of four species in Madagascar, with $M$. antsirananensis Kainul., M. atropurpureus Kainul., and $M$. maritimus Kainul. being newly described.

The molecular phylogenetic study by Kainulainen \& Bremer (2014) included samples from three of the four species recognized here, and their phylogenetic reconstruction from plastid DNA data indicates that Melanoxerus atropurpureus (as "sp. 2") and M. suavissimus are more closely related than either are to $M$. antsirananensis (as “sp. 1"). Melanoxerus maritimus was not included in that study. Kainulainen et al. (2017) estimated that the Melanoxerus clade diverged from an African ancestor around $9.4 \mathrm{Ma}$ (with a $95 \%$ highest posterior density interval of 6.6-12.4 Ma), and that the crown age, and consequently the minimum age of the dispersal of this lineage from Africa to Madagascar, is $4.2 \mathrm{Ma}$ (95\% HPD: 1.9-6.9 Ma).

\section{Taxonomic treatment}

Melanoxerus Kainul. \& B. Bremer in Taxon 63: 828. 2014.

Typus: Melanoxerus suavissimus (Homolle ex Cavaco) Kainul. \& B. Bremer ( $=$ Gardenia suavissima Homolle ex Cavaco. $\equiv$ Euclinia suavissima (Homolle ex Cavaco) J.-F. Leroy).

Deciduous or semi-deciduous shrubs or trees up to $26 \mathrm{~m}$ tall. Sympodial plagiotropic branching, usually with much reduced distal shoot internodes, terminally clustered leaves, and scarious stipules covering the terminal buds. Leaves opposite, petiolate, glossy, papyraceous to subcoriaceous, cordiform to obovate, drying dark brown to black. Stipules triangular, caducous. Flowers bisexual. Inflorescences terminal, with few or solitary flowers. Calyces shortly tubular; lobes narrowly triangular, caducous. Corollas funnelform or campanulate to broadly urceolate; lobes contorted in bud, overlapping to the left, spreading horizontally at anthesis. Styles club-shaped, ribbed, with secondary pollen presentation in the distal end. Pollen dispersed in tetrads. Fruits fleshy-indehiscent, globose to ovoid, with a thick mesocarp and an exocarp covered with conspicuous lenticels. Seeds flattened, irregularly angled, horizontally inserted and immersed in soft pulp.

Distribution, habitat and ecology. - Melanoxerus is endemic to Madagascar and distributed across most of the southern and western regions (i.e. Anosy, Androy, Atsimo-Andrefana, Menabe, Melaky, Boeny, and Sofia Regions), as well as in northernmost Madagascar in the DIANA and SAVA Regions. Melanoxerus occurs in vegetation types ranging from subarid thickets to subhumid semi-deciduous forests, from sea level to about $800 \mathrm{~m}$ in elevation, and on various substrates from coastal sands to laterite, including skeletal soils in karstic limestone formations (tsingy). It is absent from the Central Highlands and the humid east coast. Although there are exceptions, in general the species distributions reflect the ecoregions of Madagascar (cf. Olson et al., 2001), with $M$. antsirananensis being found in the dry deciduous forests of the north; $M$. atropurpureus in the inland dry deciduous forests of the west; $M$. maritimus in dry deciduous forest on coastal sands; and $M$. suavissimus in the dry spiny thicket and succulent woodlands of the southwest (Fig. 1).

Little is known about the ecology of this genus, and no information on pollination or seed dispersal has been recorded for the specimens that I have examined. The flowers are large and \pm fleshy, with funnelform to broadly urceolate corollas that are externally white (sometimes tinged purple or pale green) 
and internally purple or white with green, red, or purple markings. Pollen is usually deposited on the upper part of the style (secondary pollen presentation), which is club-shaped with longitudinal ridges. The flowers are strongly fragrant with a sweet smell like jasmine.

The fruits are large, indehiscent "berries" with a fleshy pulp and are most likely an adaptation to endozoochory. The seeds are presumably dispersed by lemurs, but it is possible that seed dispersal, at least in part, has relied on the recently extinct Malagasy megafauna, as has been suggested for Adansonia L. (BAUM, 1995) - a genus which also has large indehiscent fruits, and a distribution in Madagascar that is similar to that of Melanoxerus. The leaves of Melanoxerus appear to be attractive to herbivores because in the specimens seen in this study the leaves are usually damaged. Although not documented, insects are probably responsible for part of the damages, although slugs (pers. obs.) and lemurs of the genus Propithecus (information on the label of Meyers $\&$ Boltz 110) have been observed feeding on leaves of $M$. antsirananensis.

\section{Key to the species of Melanoxerus}

1. Leaves broadly lanceolate to obovate; flowers solitary or not; corolla tube campanulate to broadly urceolate; corolla throat glabrous; mature fruits with lenticels $1-2.5 \mathrm{~mm}$ in diam.

.. 2

1a. Leaves cordiform or ovate to lanceolate; flowers solitary; corolla tube funnelform; corolla throat pubescent; mature fruits with lenticels $0.1-1 \mathrm{~mm}$ in diam.

.. 3

2. Leaves obovate, $7-18 \times 4.5-8.5 \mathrm{~cm}$; corolla throat white with red, purple or violet (and sometimes also green) spots

1. M. antsirananensis

2a. Leaves broadly lanceolate, $10-30 \times 6.5-14.5 \mathrm{~cm}$; corolla throat uniformly purple to violet ... 2. M. atropurpureus

3. Leaves broadly ovate to cordiform; leaf length/width ratio $4: 3$ to $1: 1$; corolla lobes rounded to indented

3. M. maritimus

3a. Leaves ovate to lanceolate; leaf length/width ratio 3:1 to 3:2; corolla lobes acute 4. M. suavissimus

\section{Taxonomy}

1. Melanoxerus antsirananensis Kainul., sp. nov. (Fig. 2).

- Euclinia sp.1 in Kainulainen \& Bremer (2014: 820).

Holotypus: Madagascar. Reg. DIANA [Prov. Antsiranana]: Distr. Ambilobe, Mahamasina, Réserve

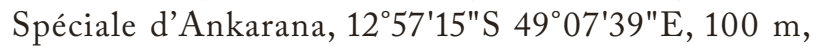
10.II.2003, fr., Bardot-Vaucoulon et al. 1406 (P [P00455719]!; iso-: K [K000551041]!, MO!, TAN).

Melanoxerus antsirananensis Kainul. differs from M. suavissimus (Homolle ex Cavaco) Kainul. \& B. Bremer by its pauciflorous inflorescences (vs. solitary flowers), campanulate (vs. funnelform) corollas, and obovate (vs. ovate to lanceolate) leaves. It most resembles $M$. atropurpureus Kainul., from which it differs in that the corolla throat is white with purple and sometimes green spots (vs. uniformly purple), and in its smaller $(7-18 \times 4.5-8.5 \mathrm{~cm}$ vs. $10-30 \times 6.5-14.5 \mathrm{~cm})$ and obovate (vs. broadly lanceolate) leaves.

Shrubs or trees up to $18 \mathrm{~m}$ tall and $40 \mathrm{~cm}$ dbh. Bark beige or whitish pale grey and smooth. Stipules 9-14.5 × 4-7 mm, triangular, interpetiolar, caducous. Leaves deciduous, petioles $0.7-6 \mathrm{~cm}$ long, adaxially canaliculate; leaf blades papyraceous, obovate, $7-18 \times 4.5-8.5 \mathrm{~cm}$; bases cuneate to attenuate, apices (rounded) obtuse to apiculate or shortly acuminate; adaxial surface: dark green when fresh, drying dark brown to black, smooth and glossy, glabrous; 8-12 pairs of conspicuous, \pm pinnate secondary veins, tertiary venation reticulate; midribs prominent, whitish green when fresh, \pm the same colour as the leaf when dry; abaxial surface green when fresh, drying dark brown to blackish, glabrous except for tuft domatia that are often present in the vein axils. Flowers solitary or few $(<10)$ in a terminal, sessile, congested and umbel-like inflorescence; pedicels up to $30 \mathrm{~mm}$ long; hypanthium narrowly urceolate, 5-8 $\times 3-4 \mathrm{~mm}$, glabrous. Calyces green; calyx tubes $0.5-2 \mathrm{~mm}$ long, externally and internally glabrous; calyx lobes $4.3-11 \times 0.8-3.5 \mathrm{~mm}$, narrowly triangular, spreading or recurved. Corollas fleshy, campanulate; corolla tubes $1.5-2.6 \mathrm{~cm}$ long, externally (yellowish) white, sometimes with purple and pale green streaks, glabrous, internally white with red, purple or violet (and sometimes also pale green) spots, glabrous or with a few trichomes (0.1-0.5 $\mathrm{mm}$ long) at the very base; corolla lobes white (sometimes with purple spots on the external side), $1-4.5 \times 0.8-2.5 \mathrm{~cm}$, ovate to deltate, rounded to acute, spreading horizontally at anthesis; stamens subsessile (filaments c. $2 \mathrm{~mm}$ long), attached $10-12 \mathrm{~mm}$ below corolla sinus and $3 \mathrm{~mm}$ from anther base; anthers $11-18 \times 1.5-2 \mathrm{~mm}$, linear, included or shortly exserted (to $2 \mathrm{~mm}$ above corolla sinus). Styles simple, clavate, with shallow longitudinal grooves, 14-20 mm long, glabrous, included or shortly exserted; stigma shortly bifid (lobes $0.5-3.5 \mathrm{~mm}$ long). Fruits (globose) ellipsoid, 3-7.6 × 2.7-6 cm, smooth, glabrous, olive to brown, with numerous beige to pale grey lenticels $(1-2 \mathrm{~mm}$ in diam.), fleshy-indehiscent with yellowish to white pulp; calyx tube sometimes persistent but calyx lobes caducous. Seeds flattened, irregularly angled, $10-15 \mathrm{~mm}$ in diam., c. $2 \mathrm{~mm}$ thick, smooth, \pm translucent, yellowish brown when fresh, drying black.

Vernacular names. - "Mamoampoma" (Rakotondrafara et al. 505); "Mantalana" (Service Forestier 8329); "Voavandrikala" (Manjakahery E Sola 83). 


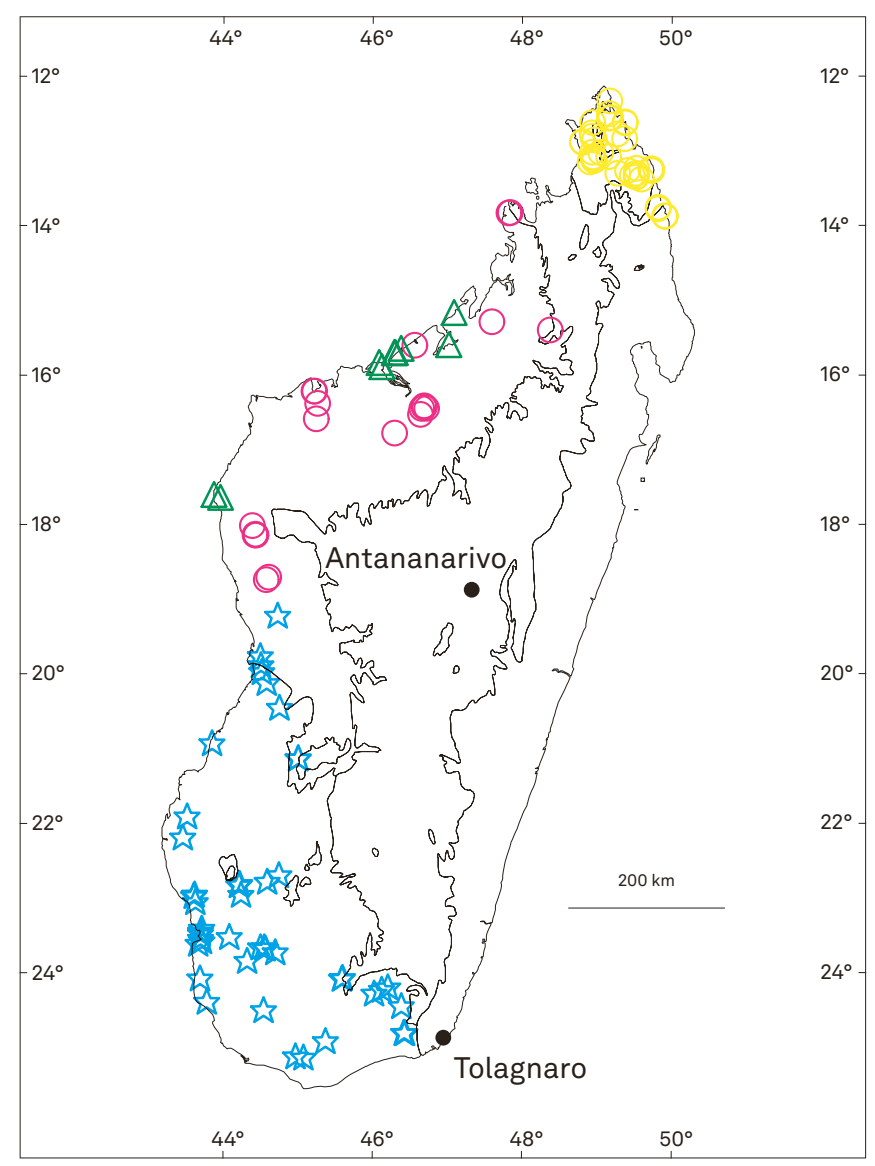

Fig. 1. - Distribution of Melanoxerus antsirananensis Kainul. (yellow circles), M. atropurpureus Kainul. (pink circles), M. maritimus Kainul. (triangles), and M. suavissimus (Homolle ex Cavaco) Kainul. \& B. Bremer (stars) in Madagascar. [mapped on the bioclimatic zones of Madagascar (after CORNET, 1974; see SCHATZ, 2000)]

Distribution, habitat and ecology. - Melanoxerus antsirananensis is only known from the DIANA and SAVA Regions in Antsiranana Province where it is widely distributed (Fig. 1). The new species grows in littoral forests and in dry deciduous forests from sea level to c. $700 \mathrm{~m}$ in elevation on Montagne d'Ambre.

Phenology. - Flowering material has been collected from September to December and fruiting material from September to July.

Conservation status. - Melanoxerus antsirananensis is known from numerous localities in northern Madagascar. Its distribution includes five protected areas, i.e. Ankarana, LokyManambato, Montagne d'Ambre, and Montagne des Français. Melanoxerus antsirananensis is therefore assigned a preliminary conservation status of "Least Concern" [LC] according the IUCN Red list Categories and Criteria (IUCN, 2012).
Notes. - The specimen Razafitsalama E Ludovic 16 is unusual in having distinctly velutinous leaves and is tentatively included here. The label on Meyers $\mathcal{E}^{2}$ Boltz 110 reports that the lemur Propithecus tattersalli eats the young leaves of this species. Melanoxerus antsirananensis flowers at the same time or just before the new leaves flush. It resembles $M$. atropurpureus in having pauciflorous inflorescences and flowers with campanulate corollas. However, the phylogenetic analysis of Kainulainen \& Bremer (2014) indicates that they are not sister species, a result that suggests that these shared characters may be plesiomorphic rather than synapomorphic.

Melanoxerus antsirananensis is distinguished from $M$. atropurpureus in its smaller leaves $(7-18 \times 4.5-8.5 \mathrm{~cm}$ vs. $10-30 \times 6.5-14.5 \mathrm{~cm})$ that are obovate rather than broadly lanceolate. Furthermore, the corolla throat is white with red to violet spots (vs. uniformly dark purple in $M$. atropurpureus).

Selected additional material examined. - Madagascar. Reg. DIANA [Prov. Antsiranana]: Masorolava, Mahagaga, Antsoroby forest, $12^{\circ} 42^{\prime} 37^{\prime \prime S}$ 4858'11"E, 53 m, 27.IX.2007, fr., Andriamihajarivo et al. 1434 (MO, P); Ankarana SR, $2 \mathrm{~km} \mathrm{~W}$ of Mahamasina village, $12^{\circ} 57^{\prime} 26^{\prime \prime S} 49^{\circ} 07^{\prime} 46 " \mathrm{E}, 132 \mathrm{~m}$, 21-30.III.1995, fr., Andrianantoanina छ Bezara 792 (BR, G, K, MO); Mon-

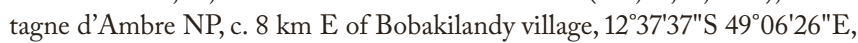
533 m, 5.XII.1995, fl., Andrianantoanina E Bezara 895 (BR, G, K, MO); Andrafiamena, forest near Anjahankely, 12 ${ }^{\circ} 54^{\prime} 23^{\prime \prime} \mathrm{S} 49^{\circ} 19^{\prime} 30^{\prime} \mathrm{E}, 450 \mathrm{~m}$, 6.I.2011, fr., Burivalova ZB 176 (G, K); Ankarana, 1250'47"S 49 06'18"E, 82 m, 14.I.2002, fr., De Block et al. 1226 (BR, MO, P, TAN, UPS, WAG); Ankarana, 4-9.III.1951, fr., Humbert \& Capuron 25493 (BR, K); ibid. loco, 1254'42"S 4906'43"E, 240-260 m, 22.V.1993, fr., Jongkind छ Rapanarivo 979 (K, MO); Montagne des Français, 12¹9'25.5"S 49²0'11"E, 230-250 m, 5.III.2016, ster., Kainulainen et al. 370 (TAN); Anosy à $4 \mathrm{~km}$ au NE du village d'Ambolobozokely [Nosy Voanio], 12²6'49"S 49³3'07"E, 22 m, 30.III.2007, fr., Rakotondrafara et al. 505 (BR, MO, P); Andranovondronina, Andakorobe, village le plus proche Antsaravy, 1208'51"S 49²0'30"E, 19 m, 17.III.2006, fr., Rakotondrafara et al. 474 (CNARP, K, MO, P, TAN); Montagne d'Ambre, 12³4'59"S 4905'59"E, 683 m, 5.II.2012, fr., Ramandimbimanana \& Randimbiarison SDR 372 (BR, G, MO); Sahafary SF, 12³5'51"S 49²6'31"E, 250 m, 7.XI.2006, fl., Ranaivojaona et al. 1551 (BR, MO, K, P, TAN); Nosy Voanio, $4 \mathrm{~km}$ E of Ambolobozokely, 12²6'01"S 49³3'00"E, $15 \mathrm{~m}$, 15.II.2006, fr., Randrianaivo et al. 1364 (BR, MO); Mosorolava, Ampombiantambo, Antsoroby forest, $12^{\circ} 42^{\prime} 13^{\prime \prime S} 48^{\circ} 58^{\prime} 12 " \mathrm{E}, 75 \mathrm{~m}, 23 . \mathrm{IX} .2007$, fl. \& fr., Ratovoson et al. 1332 (CNARP, G, MO, P, TAN); Ankarana, 11-14.I.2002, fr., Razafimandimbison $\&$ Andrianatoanina 454 (BR, MO, UPS); Ankarongana, Irodo, $\mathrm{N}$ of Analamerana forest, Ampandriantsira, 12 $39^{\prime} 42^{\prime \prime} \mathrm{S} 49^{\circ} 32^{\prime} 37^{\prime \prime} \mathrm{E}$, 50-123 m, 14.II.2001, fr., Razafitsalama \& Ludovic 16 (MO, P); Montagne des Français, 250 m, 18.II.1954, fr., Service Forestier 8329 (P, TEF); Ankarefo, 18.V.1954, fr., Service Forestier 10003 (P); Diego-Suarez, Bemaskabe forest, 200-500 m, 17.X.1927, fr., Ursch 231 (P). Reg. SAVA [Prov. Antsiranana]: Distr. Vohemar, Daraina, Bekaraoka south, $13^{\circ} 11^{\prime} \mathrm{S} 49^{\circ} 42^{\prime} \mathrm{E}$, 9.IV.2008, fr., Bremer et al. 5113 (MO, S, TAN); Hillside next to Daraina, $13^{\circ} 08^{\prime} \mathrm{S} 49^{\circ} 27^{\prime} \mathrm{E}$, 10.IV.2008, fr., Bremer et al. 5131 (MO, S, TAN); Daraina, Bobankora forest, $13^{\circ} 12.72^{\prime} \mathrm{S} 49^{\circ} 46.27^{\prime} \mathrm{E}, 200$ m, 2.II.2003, fr., Gautier et al. LG 4195 (G, K, MO); Daraina, Bekaraoka forest, $13^{\circ} 03.90^{\prime} \mathrm{S} 49^{\circ} 42.35^{\prime} \mathrm{E}, 420 \mathrm{~m}, 22 . \mathrm{XI} .2006$, fl., Gautier et al. LG 4911 (G); Nosibe, Anjiabe, Anaborano, 1304'18"S 49 54'32"E, 33 m, 11.XII.2004, fr., Manjakahery E Sola 83 (MO); Mantamena, 7 km NW of Daraina, $13^{\circ} 08^{\prime} \mathrm{S} 49^{\circ} 42^{\prime} \mathrm{E}, 112-330 \mathrm{~m}$, IV.1990, Meyers \& Boltz 110 (MO); Nosibe, Anjiabe, Analabe forest, 1304'43"S 4954'04"E, 11.V.2004, fr., Rabehevitra et al. 915 (MO, P); Tsarabaria, Manakana, Ambondrombe forest, $13^{\circ} 42^{\prime} 58^{\prime \prime S} 50^{\circ} 05^{\prime} 37 " \mathrm{E}, 3$ m, 24.X.2002, fr., Rabenantoandro et al. 1019 

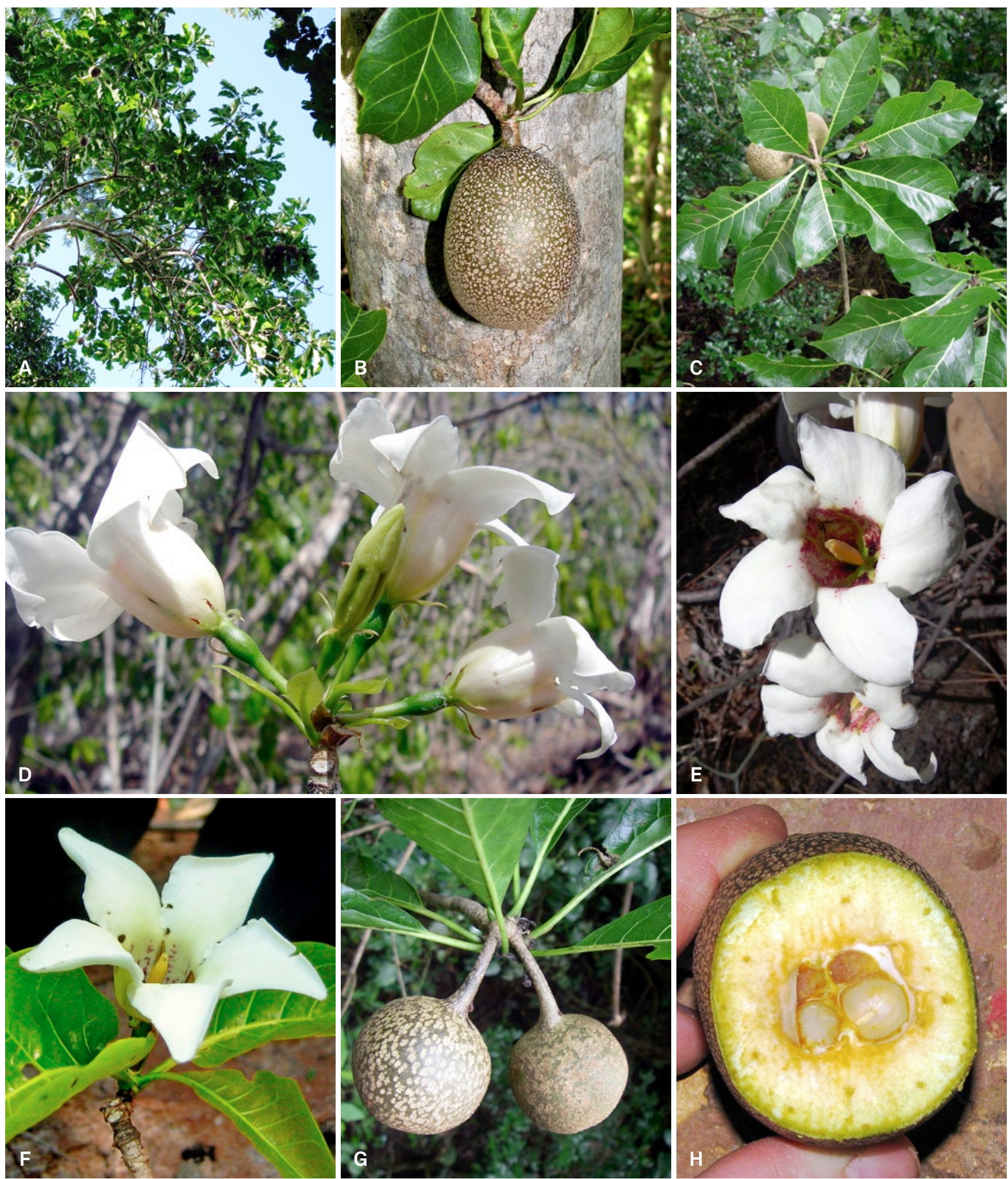

Fig. 2. - Melanoxerus antsirananensis Kainul. A. Habit; B. Fruit and smooth bark of a trunk (c. $20 \mathrm{~cm}$ dbh); C. Fruiting branch;

D-F. Flowering branch with young leaves; G. Immature fruits; H. Cross-section of a fruit showing the seeds embedded in whitish pulp.

[A-B, H: Bremer et al. 5113; C, G: Ramandimbimanana \& Randimbiarison SDR 372; D-E: Ratovoson 1332; F: Randrianaivo 1433]

[Photos: A-B, H: K. Kainulainen; C, G: S.D. Ramandimbimanana; D-E: F. Ratovoson; F: R. Randrianaivo] 
(MO); Nosibe, Anjiabe, Anaborano forest, near lake Sahaka, 130.'42"S 4954'13"E, 25 m, 2.XI.2002, fl., Rabenantoandro et al. 1091 (MO, TEF); ibid. loco, 1304'43"S 49 54'04"E, 10 m, 23.II.2003, fr., Rabenantoandro et al.

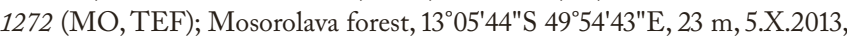
fl., Rakotovao 6482 (MO); Fanambana, Ambohimahazo forest, 1335'44"S $50^{\circ} 00^{\prime} 04 " \mathrm{E}, 60-100 \mathrm{~m}, 30 . \mathrm{III} .2001$, fr., Ranaivojaona et al. 355 (BR, MO, P); Solanampilana forest $35 \mathrm{~km} \mathrm{~N}$ of Daraina, $13^{\circ} 05^{\prime} 42^{\prime \prime S} 49^{\circ} 34^{\prime} 57^{\prime \prime} \mathrm{E}, 137 \mathrm{~m}$, 9.XII.2006, fl. \& fr., Randrianaivo et al. 1433 (G, MO, P, TAN); Daraina, Ambilondomba forest, 1309'39"S 49³8'47"E, 390 m, 31.I.2004, fr., Ranirison et al. PR 367 (G); Vohimarina, Fanambana, Antsatoby, 1336'55"S 4959'55"E, 77 m, 8.VII.2007, fr., Rasoafaranaivo 239 (MO); Analabe forest, near

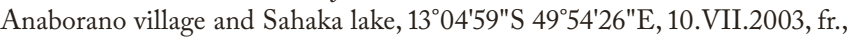
Razakamalala 519 (MO, P, TEF); Tsarabaria, Manakana, E of Ambondrobe village, $13^{\circ} 43^{\prime} 08^{\prime \prime} \mathrm{S} 50^{\circ} 05^{\prime} 50$ "E, 13.III.2004, fr., Razakamalala RZK 1022 (K, $\mathrm{MO}, \mathrm{P}, \mathrm{TEF})$.

\section{Melanoxerus atropurpureus Kainul., sp. nov. (Fig. 3).}

- Euclinia sp. 2 in Kainulainen \& Bremer (2014: 820).

Holotypus: Madagascar. Reg. Melaky [Prov. Mahajanga]: Distr. Maintirano, Beanka, partie S,

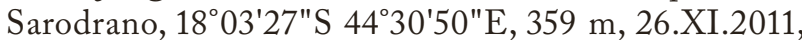
f1., Gautier, Tabinarivony \& Bolliger LG 5744 (G [G00340493]!; iso-: BR!, K [K000918390]!, MO!, P!, TEF).

Melanoxerus atropurpureus Kainul. is similar to M. antsirananensis Kainul. in its pauciflorous inflorescences and flowers with campanulate corollas, but it differs in having larger leaves $(10-30 \times 6.5-14.5 \mathrm{~cm}$ vs. $7-18 \times 4.5-8.5 \mathrm{~cm})$ that are broadly lanceolate (vs. obovate), and by the uniformly purple corolla throat (vs. whitish corolla throat with red, purple or violet and sometimes also green spots).

Shrubs or trees up to $26 \mathrm{~m}$ tall and $25 \mathrm{~cm}$ dbh. Bark grey and smooth, flaky. Stipules $2.5-13 \times 3-5 \mathrm{~mm}$, triangular, interpetiolar, caducous. Leaves deciduous, petioles $2-7.5 \mathrm{~cm}$ long, adaxially canaliculate; leaf blades papyraceous, broadly lanceolate, $10-30 \times 6.5-14.5 \mathrm{~cm}$; bases cuneate to rounded, sometimes oblique; apices acute to acuminate; adaxial surface dark green when fresh, drying dark brown to black, smooth and glossy, sparsely pubescent along venation; 8-12 pairs of conspicuous, \pm pinnate secondary veins, tertiary venation reticulate; midrib prominent, pale green when fresh, \pm the same colour as the leaf when dry; abaxial surface pale green when fresh, drying dark brown to blackish, glabrous except the pubescent veins (trichomes c. $0.1 \mathrm{~mm}$ ); tuft domatia often present in the vein axils. Flowers solitary or few $(<10)$, sessile, congested and umbel-like inflorescence; pedicels up to $15 \mathrm{~mm}$ long; hypanthium narrowly urceolate, $8-15 \times 5-8 \mathrm{~mm}$, glabrous. Calyces green; calyx tubes $2-3.5 \mathrm{~mm}$ long, externally and internally glabrous; calyx lobes 3-6.5 × 0.5-3.5 mm, narrowly triangular and recurved; corollas fleshy, campanulate to broadly urceolate; corolla tubes $1.8-4.8 \mathrm{~cm}$ long, externally white with purple and pale green streaks, glabrous, internally dark purple to violet, glabrous or with a sparse indument (trichomes $0.4-1 \mathrm{~mm}$ ) in the lowermost (c. $0.5 \mathrm{~cm}$ ) part of the tube. Corollas lobes white, $1.5-3 \times 1.2-2.5 \mathrm{~cm}$, ovate, rounded to acute, spreading horizontally at anthesis; stamens subsessile (filaments 1-4 mm long), attached $25-35 \mathrm{~mm}$ below corolla sinus and $4-7 \mathrm{~mm}$ from anther base; anthers $22-27 \times 2-3 \mathrm{~mm}$, linear, included (tips 5-6 mm below corolla sinus). Styles simple, clavate, with shallow longitudinal grooves, $28-33 \mathrm{~mm}$ long, glabrous, included; stigma shortly bifid (lobes 1-1.5 mm long). Fruits (globose) ellipsoid, 3.5-7 × 3-6 cm, smooth, glabrous, olive to brown or maroon with numerous beige to pale grey lenticels (1-2 mm in diam.), fleshy-indehiscent with yellowish to white pulp; calyx tube sometimes persistent, but lobes caducous. Seeds flattened, irregularly angled, $8-13 \mathrm{~mm}$ in diam., c. $2 \mathrm{~mm}$ thick, smooth, \pm translucent, pale yellow when fresh, drying black.

Etymology. - The specific epithet atropurpureus refers to the dark purple colour of the interior of the corolla tube in this species.

Vernacular names. - "Andrivitsy" (Réserves Naturelles 7791); "Hazondanitra" (Service Forestier 4953); "Voafotaka" (Labat E Deroin 2280); "Voangorindambo" (Service Forestier 29775).

Distribution, habitat and ecology. - Melanoxerus atropurpureus occurs in the Boeny, Melaky, and Sofia Regions (Mahajanga Province) and in the southernmost part of the DIANA Region (Antsiranana Province) (Fig. 1), where it grows in dry to humid, deciduous or evergreen forests from sea level to $450 \mathrm{~m}$ in elevation.

Phenology. - Flowering material has been collected from October to November and fruiting material from December to July.

Conservation status. - Melanoxerus atropurpureus has a wide distribution in western Madagascar and is known from several protected areas including Ankarafantsika, Bemaraha, and Namoroka. It can therefore be assigned a preliminary conservation status of "Least Concern" [LC] according the IUCN Red list Categories and Criteria (IUCN, 2012). However, it is not a commonly collected species, and more information is needed about its population size.

Notes. - The specimen Perrier de la Bâtbie $1155 A$ from the Ankara plateau is in poor shape but probably belongs to this species. In this species the exocarp of the fruit is often almost completely hidden by lenticels (Fig. 3F).

Melanoxerus atropurpureus is the tallest growing species in the genus. It mostly resembles $M$. antsirananensis but has larger leaves $(10-30 \times 6.5-14.5 \mathrm{~cm}$ vs. $7-18 \times 4.5-8.5 \mathrm{~cm})$ that are broadly lanceolate rather than obovate. Furthermore, the 

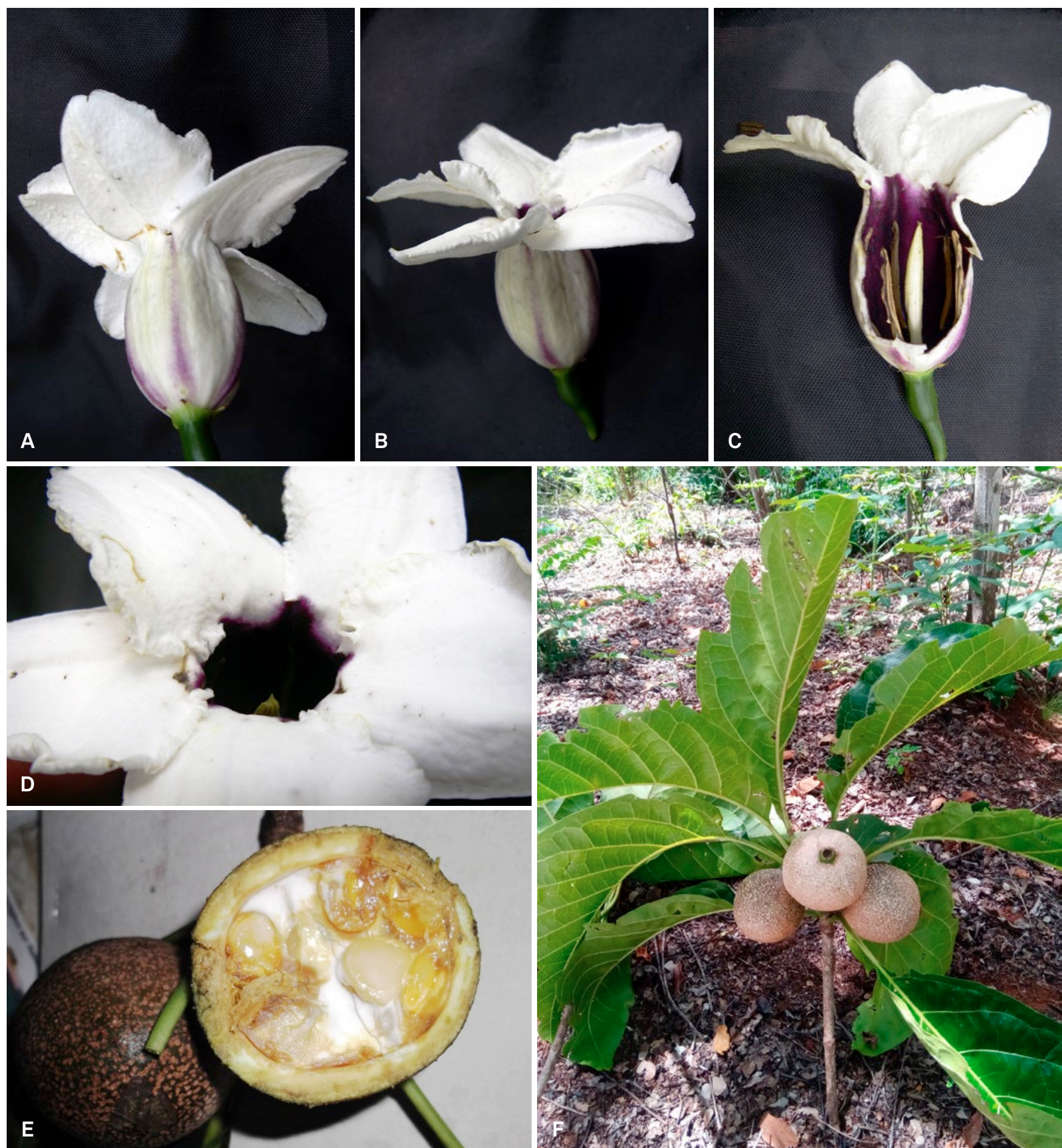

E

Fig. 3. - Melanoxerus atropurpureus Kainul. A-D. Corolla; E. Cross-section of a fruit showing the seeds embedded in whitish pulp; F. Fruiting branch. [A-D: Gautier et al. LG 5744; E: Hanitrarivo et al. HRM 146] [Photos: A-D: L. Gautier; E: M. Hanitrarivo; F: L. Rajaovelona, Ankarafantsika (Boeny), 17.III.2020] 
corolla throat is uniformly dark purple (white with red to violet spots in $M$. antsirananensis).

Selected additional material examined. - Madagascar. Reg. Boeny [Prov. Mahajanga]: Distr. Marovoay, Ankarafantsika NP, Ampijoroa forest station, 16 $18^{\prime} 52^{\prime \prime S} 46^{\circ} 49^{\prime} 04 " \mathrm{E}, 190$ m, 24.I.2000, fr., Davis et al. 2500 (K, TAN); ibid. loco, $16^{\circ} 20^{\prime} \mathrm{S} 46^{\circ} 51^{\prime} \mathrm{E}, 200 \mathrm{~m}$, 14.IV.1984, Dorr 3048 (BR, K, P, MO); ibid. loco, 1619'S 46 ${ }^{\circ} 49^{\prime} \mathrm{E}, 70-100$ m, 7.IV.1988, Gentry E Schatz 62050 (MO); Plateau d'Ankara, X.1900, fl., Perrier de la Bâthie 1155A (P); Manongarivo (Ambongo), XI.1903, fl., Perrier de la Bâtbie 1640 (P); ibid. loco, X.1905, fl., Perrier de la Bâthie 4103 (P); ibid. loco, I.1904, fr., Perrier de la Bâthie 6944 (P); Ankarafantsika, 16²0'05"S 46 47'06"E, 3-9.II.1997, fr., Rabevohitra 3027 (TEF); ibid. loco, 16¹8'S 46²4'E, 22.IV.1994, fr., Rajemisa 19 (MO, P,TAN); Distr. Mahajanga II, Mariarano, Ankatsabe forest, $15^{\circ} 28^{\prime} 30^{\prime \prime S} 46^{\circ} 41^{\prime} 24^{\prime \prime E}, 16$ m, 20.IV.2007, fr., Rakotoarivelo 94 (MO, P, TAN); Soalala, 15.VII.1977, fr., Rakotozafy 1849 (TAN); Distr. Soalala, Andranomavo, Vilanandro, Tsingy Namoroka, Ambatomay forest, $16^{\circ} 28^{\prime} 36^{\prime \prime S} 45^{\circ} 20^{\prime} 52 " \mathrm{E}, 153 \mathrm{~m}$, 9.IX.2012, fr., Ravelonarivo et al. 4523 (MO, TAN); Andranomavo, 19.XI.1955, fl., Réserves Naturelles 7791 (P, TEF); Ankarafantsika, s.d., fr., Service Forestier 19 (P); Tsingy Namoroka, 4.IV.1933, fr., Service Forestier 36 (P); sine loco, 4.IV.1933, fr., Service Forestier 54 (BR, K, P); Ankarafantsika, Ampijoroa, 15.V.1952, fr., Service Forestier 4953 (P, TEF); ibid. loco, 16.VI.1954, fr., Service Forestier 10204 (P); AmbatoBoeny, Andranovato, Ankijabe, 20.I.1959, fr., Service Forestier 19261 (TEF); Ambongo, Soalala (baie de Baly), 22.XI.1965, fl., Service Forestier 24244 (BR, P,TEF); by lake Ampijoroa, 28.II.1980, fr., Service Forestier 29775 (TEF). Reg. DIANA [Prov. Antsiranana]: Distr. Ambanja, Ampasindava, Andranomatavy forest, 1340'04"S 47'59'21"E, 295 m, 25.XI.2009, fl., fr., Madiomanana et al. MAD 266 (G, K, MO, P, TEF); along Andranomatavy river, 1340'39.19"S 4758'30.24"E, 260 m, 2.VII.2012, fr., Tabinarivony \& Rasoanaivo TAJ 699 (G). Reg. Melaky [Prov. Mahajanga]: Beanka, Ambinda-Nord, $17^{\circ} 55^{\prime} 51^{\prime \prime S}$ 442'이"E, 216 m, 5.II.2012, fr., Hanitrarivo et al. HRM 146 (G, S); Ambodiria, $1 \mathrm{~km}$ E of Ambinda (RN 9, near Antsalova), $18^{\circ} 38^{\prime} \mathrm{S} 44^{\circ} 42^{\prime} \mathrm{E}, 100-200 \mathrm{~m}$, 3.XII.1992, fr., Labat \& Deroin 2280 (K, P, TAN); Beanka, Sarodrano, 1802'55"S 4431'13"E, 446 m,11.XII.2011, fl., Nusbaumer et al. LN 3073 (G, K); Antsalova, 20.III.1993, fr., Villiers et al. 4851 (MO, P); Belitsaka, Ankilimanarivo, Beanka forest, $18^{\circ} 03^{\prime} 37^{\prime \prime} \mathrm{S} 44^{\circ} 31^{\prime} 37^{\prime \prime} \mathrm{E}, 319 \mathrm{~m}, 16 . I V .2011$, fr., Razakamalala E Germain 6382 (MO, S). Reg. Sofia [Prov. Mahajanga]: entre Antsohihy et Befandriana-Nord, X.1962, fl., Bosser 16697 (P, TAN); Distr. Antsohihy,

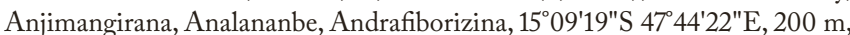
30.V.2000, fr., Rakotonasolo RNF 177 (BR, K, P, TAN).

3. Melanoxerus maritimus Kainul., sp. nov. (Fig. 4).

Holotypus: Madagascar. Reg. Melaky [Prov. Mahajanga]: Distr. Maintirano, Lac Mandrozo, 17'32'43"S 4402'26"E, 18 m, 23.XI.2015, fl. \& fr., Gautier, Luino E Rasolonjatovo LG 6196 (G [G00419027]!; iso-: MO!, P!, S!, TEF).

Melanoxerus maritimus Kainul. differs from M. suavissimus (Homolle ex Cavaco) Kainul. E B. Bremer in its rounder leaves (leaflength/width ratio 4:3-1:1 vs. 3:1-3:2), that have a truncate to cordate leaf base (vs. cuneate to attenuate), and corolla lobes that are rounded to indented (vs acute).

Shrubs or trees up to $10 \mathrm{~m}$ tall and $28 \mathrm{~cm}$ dbh, with mostly sympodial branching. Bark pale grey to pale brown, smooth, flaky. Stipules $3.5-4.7 \times 3.6-4.5 \mathrm{~mm}$, broadly triangular, interpetiolar, caducous. Leaves semi-deciduous, petioles $2.8-5.2 \mathrm{~cm}$ long, adaxially canaliculate; leaf blades papyraceous to subcoriaceous, cordiform to broadly ovate, $7.5-12.7 \times 5-10.5 \mathrm{~cm}$; bases cordate to truncate; apices acute; adaxial surface dark green when fresh, drying dark brown to black, smooth and very glossy, glabrous except for the lower part of the midrib (trichomes $0.1-0.5 \mathrm{~mm}$ long); 6-9 pairs of conspicuous, \pm pinnate secondary veins, tertiary venation reticulate; midrib prominent, pale green when fresh, \pm the same colour as the leaf when dry; abaxial surface green when fresh, drying dark brown to blackish, glossy, glabrous, but with tuft domatia often present in the vein axils. Flowers solitary, terminal, very fragrant; pedicels up to $14 \mathrm{~mm}$ long; hypanthium narrowly urceolate, 5-18 $\times 3-4 \mathrm{~mm}$, glabrous; calyces green; calyx tubes $1-4.5 \mathrm{~mm}$ long, externally and internally glabrous; calyx lobes 3.4-6.2 ×1.2-2 mm, narrowly triangular and recurved; corollas fleshy, funnelform, straight; corolla tubes 2-3.7 cm long, externally pale yellow with a greenish base, glabrous, internally white with red spots and lines, finely pubescent (trichomes c. $0.5 \mathrm{~mm}$ long). Corollas lobes white, $2-2.7 \times 1.6-3 \mathrm{~cm}$, ovate to orbicular, rounded to truncate or indented, spreading horizontally at anthesis; stamens subsessile (filaments $2-4 \mathrm{~mm}$ long), attached c. $10 \mathrm{~mm}$ below corolla sinus and c. $4 \mathrm{~mm}$ from anther base; anthers c. $15 \times 2 \mathrm{~mm}$, linear, exserted for 1-3 mm. Styles simple, clavate, with shallow longitudinal grooves, 30-35 mm long, glabrous, exserted for 5-9.5 mm; stigma shortly bifid (lobes c. $3 \mathrm{~mm}$ long). Fruits ellipsoid, 2.5-5.1 × 1.6-3.7 cm, smooth, glabrous, (colour of mature fruit unknown) with numerous beige lenticels (0.1-1 mm in diam.), fleshy-indehiscent; calyx tube persistent but lobes caducous. Seeds not seen.

Etymology. - This species grows near the sea and that is what the specific epithet maritimus refers to.

Vernacular names. - "Ndraniana" (Service Forestier 10585); "Tselitselika" (Bertrand 16).

Distribution, habitat and ecology. - Melanoxerus maritimus is known from sea level to $30 \mathrm{~m}$ in elevation in coastal areas of the Boeny, Melaky, and Sofia Regions (Mahajanga Province) (Fig. 1). It grows in low canopy, dry deciduous forests on dunes and on edges of salt flats or mangroves.

Phenology. - Flowering material has been collected from June to November, and fruiting material from July to February.

Conservation status. - Melanoxerus maritimus is known from less than ten locations, (three of which are within the recently protected areas of Antrema, Bombetoka Belemboka, and Mandrozo), with an estimated area of occupancy (AOO) of less than $2000 \mathrm{~km}^{2}$. It appears restricted to littoral dry forest. This habitat is impacted for firewood or charcoal production (also noted on label of De Block \& Rakotonasolo 

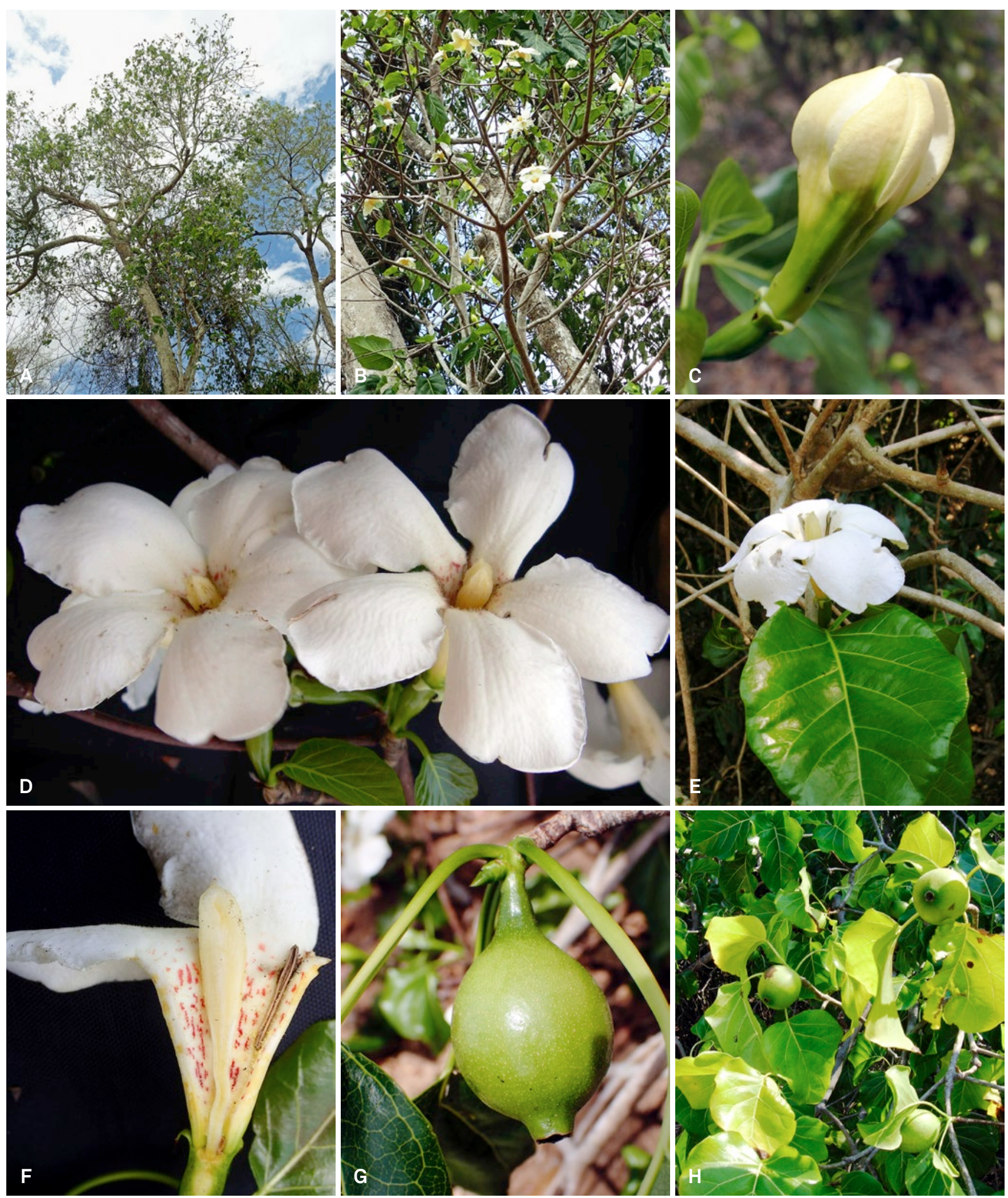

Fig. 4. - Melanoxerus maritimus Kainul. A. Habit; B. Flowering branch; C. Flower bud; D-E. Open flowers;

F. Transverse section of the corolla and stigma; G-H. Immature fruits. [A-C, F-G: Gautier et al. LG 6196; D: Randriatsivery 207;

E: Labat et al. 3534; H: Labat et al. 3535] [Photos: A-C, F-G: L. Gautier; D: M. Randriatsivery; E, H: J.-N. Labat] 
806). It is projected that the number of locations and the number of mature individuals continue to decline, and this species can therefore be assigned a preliminary conservation status of "Vulnerable"[VUB2ab(iii)] according to IUCN Red list Categories and Criteria (IUCN, 2012). However, more information is needed about the population size of this species.

Notes. - Melanoxerus maritimus has solitary flowers with a funnelform corolla and pubescent corolla throat, and in these characters it most resembles $M$. suavissimus, but it is recognized by its \pm cordiform leaves that are almost as wide as long (leaf length/width ratio 4:3-1:1 vs. 3:1-3:2 in $M$. suavissimus), and by having corolla lobes that are rounded to indented rather than acute. The leaves of $M$. maritimus are glossy below and very glossy above. They remain glossy when dried and almost appear varnished. It is notable that none of the specimens examined in this study is lacking leaves, and that specimens collected during the dry season still retain some leaves. This species is therefore probably best considered semi-deciduous as opposed to deciduous.

Selected additional material examined. - Madagascar. Reg. Boeny [Prov. Mahajanga]: [Distr. Mahajanga I] Antananamasadza [probably Antanimasaja, now part of Mahajanga town], 1912, Afzelius 266 (P, S); Katsepy, c. 10 m, 19.II.1950, ster., Bertrand 16 (P); Distr. Mahajanga II, Antsanitia, Belamonty forest, 15³4'19"S 46²5'04"E, 9 m, 14.II.1999, fr., De Block \& Rakotonasolo 806 (BR, MO, TAN); Katsepy, 1545'56"S 46²1'21"E, 0 m, 22.VII.1995, fl. \& fr., Edmondson et al. 95-17 (MO, P); ibid. loco, 8.IX.1912, fl., Kaudern s.n. (S); ibid. loco, $15^{\circ} 42^{\prime} 35^{\prime \prime S} 46^{\circ} 12^{\prime} 03^{\prime \prime E}, 10 . X I .2005$, fl., Labat et al. 3534 (K, P); ibid. loco, 10.XI.2005, fr., Labat et al. 3535 (G, K, MO, P); Antsanitia, 15³4'31"S $46^{\circ} 25^{\prime} 7 " \mathrm{E}, 30$ m, 7.I.2000, fr., Rakotonasolo RNF 132 (K, TAN); Forest station E of Antsanitia village, 1534'31"S 46 24'26"E, 16 m, 15.II.2013, fr., Ralimanana et al. 1803 (BR, K, MO, P, TAN); Distr. Mahajanga II, Ampasimariny, 9 m, 23.VIII.1954, fl. \& fr., Service Forestier 10585 (MO, P, TEF). Reg. Melaky [Prov. Mahajanga]: Region de Tambohorano, 23.VI.1930, fl., Decary 8083 (P); Ambongo, 1841, fr., Pervillé 673 (K, P). Reg. Sofia [Prov. Mahajanga]: SW of Antangena, $15^{\circ} 28^{\prime} \mathrm{S} 47^{\circ} 09^{\prime} \mathrm{E}, 0$ m, 25.X.1987, fl. \& fr., Bisset M12 (K); Distr. Analalava, Antonibe, 1501'44"S 47 13'17"E, 19.VIII.2007, fl. \& fr., Randriatsivery 207 (MO, P, TAN).

4. Melanoxerus suavissimus (Homolle ex Cavaco) Kainul. \& B. Bremer (Fig. 5).

$\equiv$ Gardenia suavissima Homolle ex Cavaco in Adansonia ser. 2, 7: 177.1967. E Euclinia suavissima (Homolle ex Cavaco) J.-F. Leroy in Adansonia ser. 2, 14: 52. 1974.

Holotypus: Madagascar. Reg. Androy [Prov. Toliara]: Ambovombe, 27.X.1924, Decary 3357 (P [P00852562]!).

Shrubs or trees up to $13 \mathrm{~m}$ tall and $40 \mathrm{~cm}$ dbh, with mostly sympodial branching. Bark (mottled) whitish or pale grey to pale brown, smooth, flaky. Stipules 3-10 × 2-4 mm, triangular, interpetiolar, caducous. Leaves deciduous, petioles $0.5-5 \mathrm{~cm}$ long, adaxially canaliculate; leaf blades papyraceous, ovate to lanceolate, $6.5-16 \times 2-9(-11) \mathrm{cm}$; bases (rounded) cuneate to attenuate (often asymmetrically); apices acute to acuminate; adaxial surface dark green when fresh, drying dark brown to black, smooth, matte or glossy, \pm glabrous to (velvety) pubescent in particular along midrib and venation; $(6-) 8-10(-12)$ pairs of conspicuous, \pm pinnate secondary veins, tertiary venation reticulate; midribs prominent, pale green when fresh, \pm the same colour as the leaf when dry; abaxial surface pale green when fresh, drying dark brown to blackish, matte or glossy, \pm glabrous or with pubescent midrib and venation, tuft domatia often present in the vein axils. Flowers solitary, terminal, very fragrant; pedicels up to $9 \mathrm{~mm}$ long; hypanthium narrowly urceolate, $4.5-12 \times 2.5-5.5 \mathrm{~mm}$, glabrous; calyces green; calyx tubes $1-5.5 \mathrm{~mm}$ long, externally and internally glabrous; calyx lobes 4.5-8 $\times 1-3 \mathrm{~mm}$, narrowly ovate, acuminate, recurved; corollas fleshy, funnelform, straight; corolla tubes $2-4.5 \mathrm{~cm}$ long, externally white to pale yellow often with a greenish base, glabrous, internally white with pink, red, or purple spots, finely pubescent (trichomes $0.5-1.5 \mathrm{~mm}$ long). Corollas lobes white (sometimes pale yellow on the external side), $2-4.5 \times 1-3 \mathrm{~cm}$, ovate, acute, spreading horizontally at anthesis; stamens subsessile (filaments c. $1 \mathrm{~mm}$ long), attached $10-14 \mathrm{~mm}$ below corolla sinus and $6-10 \mathrm{~mm}$ from anther base; anthers $22-26 \times 1.5-2 \mathrm{~mm}$, linear, exserted for c. $5 \mathrm{~mm}$. Styles simple, clavate, with shallow longitudinal grooves, to c. $5 \mathrm{~cm}$ long, glabrous, usually shortly exserted for c. $5 \mathrm{~mm}$; stigma shortly bifid (lobes c. $5 \mathrm{~mm}$ long). Fruits globose to ellipsoid, 3-5 × 2.5-3.5 cm, smooth, glabrous, olive to grey brown or dark purple to black with numerous beige to pale grey lenticels $(0.1-1 \mathrm{~mm}$ in diam.), fleshy-indehiscent; calyx tube usually persistent, but lobes caducous. Seeds flattened, irregularly angled, $7-10 \mathrm{~mm}$ in diam., c. $2 \mathrm{~mm}$ thick, smooth, yellowish brown when fresh, drying black.

Vernacular names. - "Asomitsoy" (Rakotonasolo et al. RNF 932); "Hazomfotaka" (Réserves Naturelles 6762); "Kaliona" (Koechlin s.n.); "Mahabiboala" (Service Forestier 29830); "Vala" (Service Forestier 5797); "Voafotaka" (Decary 4556); "Volatsiva" (Service Forestier 4136).

Distribution, habitat and ecology. - Melanoxerus suavissimus is widespread in subarid vegetation in the Androy, Anosy, Atsimo-Andrefana, and Menabe Regions (Toliara Province), and occurs as far north as the Manambolo river in the southernmost part of the Melaky Region (Mahajanga Province) (Fig. 1). It is found from sea level to c. $800 \mathrm{~m}$ in elevation.

Phenology. - Flowering specimens have been collected in (April) July to December, and fruiting specimens throughout the year.

Conservation status. - Melanoxerus suavissimus is widespread in south-western Madagascar and has been assigned an IUCN conservation status of "Least Concern" [LC] (IUCN, 2021). 

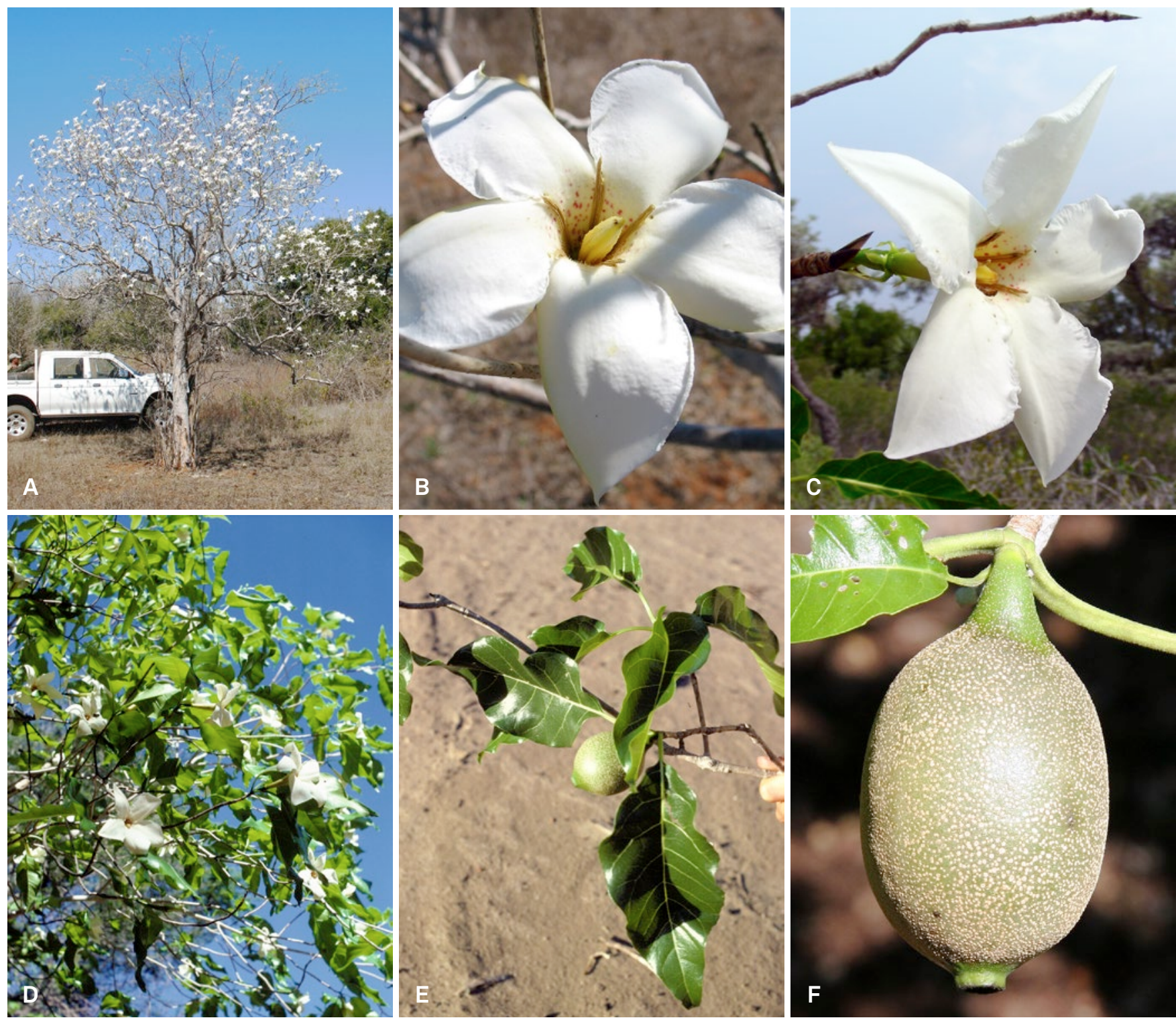

Fig. 5. - Melanoxerus suavissimus (Homolle ex Cavaco) Kainul. \& B. Bremer. A. Habit and habitat; B. Top view of the flower; C. Flower; D. Flowering branch; E-F. Immature fruits. [A-B: Ranaivojaona et al. 1456; D: Phillipson 2413; E-F: Andriamihajarivo et al. 1814] [Photos: A-B, D-E: P. Phillipson; C: S. Landrein, Ifaty (Toliara), 5.X.2006; F: K. Kainulainen]

Notes. - Since Cavaco's (1967) original description of Melanoxerus suavissimus (as Gardenia suavissima) included specimens of both $M$. atropurpureus and M. maritimus, an amended description is provided.

This is the most widespread and the most commonly collected species. However, few collections have been made in from May to August (none from June), and presumably this species is usually leafless during that time. Melanoxerus suavissimus usually flowers at the same time or just before the new leaves flush (cf. Fig. 5A, D). Apparently, the juice of the fruits turns black and is used for making tattoos (SсHATZ, 2001). Information about of the use of Melanoxerus by local people is otherwise scant, although according to the label information on the specimen Ranaivojaona et al. 248, the fruits are edible.

Selected additional material examined. - Madagascar. Reg. Androy [Prov. Toliara]: Besakoa, 2456'36"S 45²7'59"E, 227 m, 4.III.2003, fr., Andriamahay 497 (K); Imanombo, XI.1952, fr., Bosser 3787 (P); Beraketa, X.1956, fl., Bosser 10237 (P); Ambovombe, XII.1959, fl. \& fr., Bosser 13353 (P, TAN); Beraketa, on road to Isoanala, $10 \mathrm{~km} \mathrm{~S}$ of Soanala, 646 m,19.XII.2004, fr., Rakotonasolo et al. RNF 932 (K, TAN); Antanimora, 30.VII.1926, fr., Decary 4556 (P); Behara, 22.X.1954, fl., Réserves Naturelles 6762 (P); Ampandrandava, 700-800 m, X.1942, fl., Seyrig 122 (P); Beloha, 19.II.1949, fr., Service Forestier 454 (P, TEF); Imanombo-Ifotaka (rte), 26.IX.1952, fr., Service Forestier 5797 (P, TEF). Reg. Anosy [Prov. Toliara]: Distr. Amboasary Sud, Marotsiraka, 


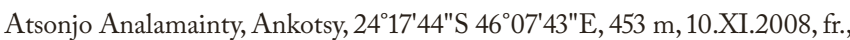
Andriamibajarivo et al. 1527 (G, MO); Anadabolava, 200-250 m, XII.1933, fr., Humbert 12450 (P); Andohahela, Ihazofotsy, 2450'S 46³2'E, 100 m, 16.IV.1996, fr., Laha 75 (BR, G, K, MO); Fort Dauphin, Analamatahitra, 31.I.1952, fr., Service Forestier 4463 (P, TEF); Fort Dauphin, Anteviala, 10.IV.1960, fr., Service Forestier 10789 (P, TEF). Reg. Atsimo-Andrefana [Prov. Toliara]: Distr. Sakaraha, forêt d'Analaraty, 22 49'14"S 44¹7'16"E, 520 m, 20.XII.2010, fr., Andriamihajarivo et al. 1814 (MO, P, TAN);

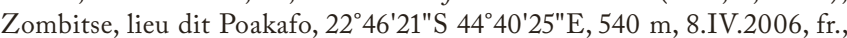
Andriamihajarivo et al. 905 (MO, TAN); Befandriana-Sud, 150 m, 9.IX.1961, fl. \& fr., Appert 203 (MO, Z); along rte \#10 between Betioky and Ejeda, 175-225 m, 14.II.1975, fr., Croat 31241 (MO, TAN); St. Augustin, 2328'58"S 4345'58"E, 0 m, 6.I.1999, fr., De Block et al. 561 (BR, K, MO, P, TAN); road from Betioky to Ampanihy, c. $23 \mathrm{~km}$ after Ejeda, 24 $31^{\prime} 17^{\prime \prime} \mathrm{S} 44^{\circ} 37^{\prime} 25^{\prime \prime} \mathrm{E}, 244 \mathrm{~m}$, 6.II.2007, fr., De Block et al. 2323 (BR, MO, P, S, TAN, WAG); entre Tongobory et aux environs d'Ambovombe, 117-214 m, 20.I.2004, fr., Hong-Wa et al. 178 (G, MO); forêt de Besomaty entre le Fiherenana et l'Isahaina (Mangoky), 750-800 m, X.1933, fl., Humbert 11250 (P); Manombo, Fiherenamasay, 18.XI.1967, fl., Koechlin s.n. (P); St. Augustin, 2327' S 4346' E, 10 m, 1.II.1990, fr., Labat \& Du Puy 2045 (K, MO, P); vallée du Fiharenana, 0-200 m, 9.XI.1960, fl. \& fr., Leandri 3778 (P); lac Tsimanampetsotsa, 0-100 m, 23.XI.1960, fr., Leandri \&' Saboureau 4451 (BR, K, P); 20-30 km N of Tulear on the road to Morombe, $23^{\circ} 12^{\prime} \mathrm{S} 43^{\circ} 37^{\prime} \mathrm{E}, 5^{-10} \mathrm{~m}, 11 . \mathrm{XI} .1989$, fl. \& fr., Miller E Keating 4513 (K, MO, P, TAN); rte d'Ankazoabo, II.1967, fr., Morat 2455 (P, TAN); vallée de Fiherenana, X.1924, fl., Perrier de la Bathie 16653 (P); Beza Mahafaly Reserve near Betioky, 2340'S 443'ㄹ, 160 m, 17.V.1987,

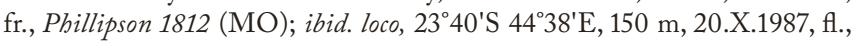

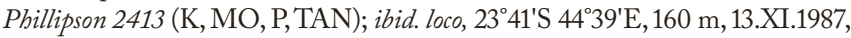
fr., Phillipson 2558 (K, MO, P, TAN); Itampolo, Montagne de Haktokaliotse, 242ㄴ'26"S 4351'01"E, 100 m, 1.IX.1998, fr., Rakotomalaza \& Messmer 1653 (G, K); Ambohimahavelona, Sept Lacs, Andranolahy, 2331'32"S 4409'27"E, 100 m, 15.IX.1998, fl., Rakotomalaza \& Messmer 1725 (G, K); Manombo, near PK 32, 2303'00"S 4341'42"E, 9.XII.2004, fr., Rakotonasolo et al. RNF 880 (BR, K, MO, TAN); Basibasy, Mikea forest, 22 ${ }^{\circ} 10^{\prime} 13^{\prime \prime S} 43^{\circ} 31^{\prime} 30 " \mathrm{E}, 100 \mathrm{~m}$, 31.I.2000, fr., Ranaivojaona et al. 248 (G, K, MO); Belalanda, Ranobe, $22^{\circ} 56^{\prime} 49^{\prime \prime S} 43^{\circ} 41^{\prime} 08^{\prime \prime E}, 135$ m, 15.III.2006, fr., Ranaivojaona et al. 1425 (P);

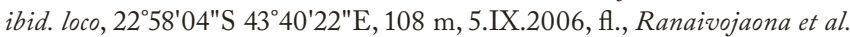
1456 (G, K, MO, P); Sakaraha, Mahaboboka, Ambinanintelo, Forêt de Satra, 22 48'10"S 44'17'54"E, 470 m, 23.III.2013, fr., Randrianarivony et al.

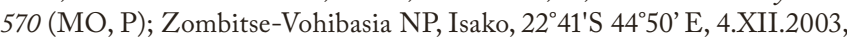
ster., Razafimandimbison \& Bremer 496 (MO, TAN, UPS); Beroroha, Antsoa, Androtsy forest, $21^{\circ} 05^{\prime} 43^{\prime \prime S} 45^{\circ} 05^{\prime} 48^{\prime \prime E}, 254$ m, 9.I.2011, fr., Razakamalala 5851 (MO); Imonty, Ankazoabo, Sakaraha, 27.XI.1951, fr., Service Forestier 4136 (P, TAN, TEF); Antseva, Besifake, 500 m, 11.II.1955, fr., Service Forestier 12815 (P, MO, TEF); Sarodrano, XII.1961, fl., Service Forestier 20830 (BR, K, MO, P, TEF). Reg. Melaky [Prov. Mahajanga]: Tsingy de Bemaraha, $19^{\circ} 09^{\prime} \mathrm{S} 44^{\circ} 49^{\prime} \mathrm{E}, 50 \mathrm{~m}, 10 . X I I .1996$, fr., Jongkind et al. 3482 (BR, K, L, MO, TAN). Reg. Menabe [Prov. Toliara]: village avant la Réserve d'Andranomena, 17.X.1999, fl., Allorge 2428 (P); on the road to Belo, c. $68.5 \mathrm{~km} \mathrm{NE}$ of Morondava, $19^{\circ} 55^{\prime} 06^{\prime \prime S} 44^{\circ} 36^{\prime} 00^{\prime E} \mathrm{E}, 5 \mathrm{~m}$, 22.II.2000, fr., Davis et al. 2587 (BR, K, MO, TAN); Kirindy Mitea NP, 20 53'14"S 4355'22"E, 20 m, 21.III.2011, fr., Razafimandimbison छ Kainulainen 1205 (MO, S); N de Tsimafana env. 2 km, 25.V.1980, fr., Service Forestier 29830 (TEF).

\section{Ackowledgements}

I thank the curators of G, K, MO, P, S, TAN, TEF, and UPS for providing access to their collections, and Laurent Gautier, Mitia Hanitrarivo, the late Jean-Noël Labat, Sven Landrein, Peter Phillipson, Landy Rita Rajaovelona, Solotiana D. Ramandimbimanana, Richard Randrianaivo, Monique Randriatsivery,
Fidisoa Ratovoson, Désiré Ravelonarivo, and A. Jacquis Tahinarivony for providing photographs. Conservatoire et Jardin botaniques de Genève, Muséum national d'Histoire naturelle, and Missouri Botanical Garden have also made photographs available. I also thank Petra De Block, Laurent Gautier, Sylvain Razafimandimbison, and Martin Callmander for helpful comments to improve the manuscript.

\section{References}

Baum, D. (1995). A systematic revision of Adansonia (Bombacaceae). Ann. Missouri Bot. Gard. 82: 440-471.

Cavaco, A. (1967). Un nouveau Gardenia (Rubiacées) de Madagascar. Adansonia ser. 2, 7: 177-179.

Cornet, A. (1974). Essai de cartographie bioclimatique à Madagascar. Notice Explicative ORSTOM 55.

IUCN (2012). IUCN Red List Categories and Criteria: Version 3.1. Ed. 2. IUCN Species Survival Commission, Gland and Cambridge.

IUCN (2021). The Red List of threatened species. [https://www.iucnredlist.org]

Kainulainen, K. \& B. Bremer (2014). Phylogeny of Euclinia and allied genera of Gardenieae (Rubiaceae), and description of Melanoxerus, an endemic genus of Madagascar. Taxon 63: 819-830. DOI: https://doi.org/10.12705/634.2

Kainulainen, K., S.G. Razafimandimbison, N. Wikström \& B. Bremer (2017). Island hopping, long-distance dispersals and species radiations in the Western Indian Ocean: historic biogeography of the Coffeeae alliance (Rubiaceae). J. Biogeogr. 44: 1966-1979. DOI: https://doi.org/10.1111/jbi.12981

Leroy, J.-F. (1974). Recherches sur les Rubiacées de Madagascar: les genres Mantalania et Pseudomantalania (Gardéniées). Adansonia ser. 2, 14: 29-52.

Madagascar Catalogue (2021). Catalogue of the plants of Madagascar. Missouri Botanical Garden, St. Louis \& Antananarivo. [http://www.tropicos.org/Project/Madagascar]

Olson, D.M., E. Dinerstein, E.D. Wikramanayake, N.D. Burgess, G.V.N. Powell, E.C. Underwood, J.A. D'Amico, I. Itoua, H.E. Strand, J.C. Morrison, C.J. Loucks, T.F. Allnutt, T.H. Ricketts, Y. Kura, J.F. Lamoreux, W.W. Wettengel, P. Hedao \& K.R. Kassem (2001). Terrestrial ecoregions of the world: a new map of life on Earth. BioScience 51: 933-938.

Schatz, G.E. (2000). Endemism in the Malagasy tree flora. In: Lourenço, W.R. \& S.M. Goodman (ed.), Diversity and Endemism in Madagascar: 1-9. Mémoires de la Société de Biogéographie, Paris.

Schatz, G.E. (2001). Generic tree flora of Madagascar. Royal Botanic Gardens, Kew. 